Thaís Rocha Assis ${ }^{1}$

Ana Claudia Antonio Maranhão Sáz

Waldemar NAVES DO AMARAL ${ }^{3}$

Elicéa Marcia Batista ${ }^{2}$

Cibele Kayenne Martins Roberto Formiga

Délo Marques Conde

Artigo Original

Palavras-chave

Terapia por exercícios Contração muscular/fisiologia

Força muscular

Diafragma da pelve

Período pós-parto

Atenção primária à saúde

Keywords

Exercise therapy

Muscle contraction/physiology

Muscle strength

Pelvic floor

Postpartum period

Primary health care

\title{
Efeito de um programa de exercícios para o fortalecimento dos músculos do assoalho pélvico de multíparas
}

\author{
The effect of an exercise program to strengthen pelvic floor muscles \\ in multiparous women
}

Resumo

OBJETIVOS: Investigar o efeito de um programa individualizado e supervisionado de exercícios para os músculos do assoalho pélvico (MAP) no pós-parto de multíparas e verificar a correlação entre dois métodos de medida de força dos MAP. MÉTODOS: Conduziu-se um ensaio clínico aberto em que foram incluídas puérperas, multíparas com idade entre 18 e 35 anos. A amostra foi de 23 puérperas divididas em dois grupos: Grupo Intervenção (Gl, n= 1 1) e Grupo Controle (GC, n= 12). As puérperas do GI participaram de um programa de exercícios para os MAP durante oito semanas, com frequência de duas vezes por semana. As puérperas do GC não receberam orientação quanto à prática de exercícios. A força dos MAP foi medida em duas oportunidades, utilizando-se a palpação vaginal digital e o perineômetro. A análise estatística foi realizada através dos seguintes testes: exato de Fisher, do $\chi^{2}$, $t$ de Student, Kolmogov-Smirnov para duas amostras e coeficiente de correlação de Pearson. Foi considerado como significativo $p<0,05$. RESULTADOS: A média de idade das participantes do $G$ foi de $24 \pm 4,5$ anos e do $G C$ foi de $25,3 \pm 4$ anos $(p=0,4)$. Após o programa de exercícios, verificou-se diferença significativa entre os grupos nas duas medidas da força muscular ( $p<0,001)$. Os dois métodos de medida da força muscular apresentaram correlação significativa nas duas avaliações $\left(1^{a}\right.$ avaliação: $r=0,889, p<0,001 ; 2^{a}$ avaliação: $\left.r=0,925, p<0,001\right)$. CONCLUSÕES: $\bigcirc$ programa de exercícios resultou em aumento significativo da força dos MAP. Verificou-se boa correlação entre a palpação vaginal digital e o perineômetro, indicando que a palpação vaginal pode ser utilizada na prática clínica por ser um método de baixo custo e que demonstrou uma correlação significativa com um método objetivo, o perineômetro.

\section{Abstract}

PURPOSES: To investigate the effect of an individualized and supervised exercise program for the pelvic floor muscles (PFM) in the postpartum period of multiparous women, and to verify the correlation between two methods used to assess PFM strength. METHODS: An open clinical trial was performed with puerperal, multiparous women aged 18 to 35 years. The sample consisted of 23 puerperal women divided into two groups: Intervention Group (IG, n= 1 1) and Control Group (CG, n= 12). The puerperal women in IG participated in an eight-week PFM exercise program, twice a week. The puerperal women in CG did not receive any recommendations regarding exercise. PFM strength was assessed using digital vaginal palpation and a perineometer. The statistical analysis was performed using the following tests: Fisher's exact, $\chi^{2}$, Student's $t$, Kolmogorov-Smirnov for two samples, and Pearson's correlation coefficient. Significance was defined as $p<0.05$. RESULTS: The participants' mean age was $24 \pm 4.5$ years in IG and $25.3 \pm 4$ years in CG $(p=0.4)$. After the exercise program, a significant difference was found between the groups in both modalities of muscle strength assessment $(p<0.001)$. The two muscle strength assessment methods showed a significant correlation in both assessments $\left(1^{\text {st }}\right.$ assessment: $r=0.889, p<0.001 ; 2^{\text {nd }}$ assessment: $\left.r=0.925, p<0.001\right)$. CONCLUSIONS: The exercise program promoted a significant improvement in PFM strength. Good correlation was observed between digital vaginal palpation and a perineometer, which indicates that vaginal palpation can be used in clinical practice, since it is an inexpensive method that demonstrated significant correlation with an objective method, i.e. the use of a perioneometer.

Registrado no ReBEC (Registro Brasileiro de Ensaios Clínicos) sob o número RBR-9hhr2g.

Correspondêncio

Thaís Rocha Assis

Universidade Federal de Goiás - Campus Jatai Rodovia BR 364, Km 192 - Parque Industrial (EP: $75801-615$ Jataí (GO), Brasil

Recebido

$25 / 07 / 2012$

Aceito com modificacōõs

$14 / 09 / 2012$
Trabalho realizado na Maternidade Pública e Unidades Básicas de Saúde - Jataí (GO), Brasil.

'Departamento de Fisioterapia, Universidade Federal de Goiás - UFG - Goiânia (GO), Brasil.

2Departamento de Fisioterapia, Enfermagem e Nutrição da Pontifícia, Universidade Católica de Goiás - PUC Goiás - Goiânia (GO), Brasil. 3Programa de Pós-graduação em Ciências da Saúde, Universidade Federal de Goiás - UFG - Goiânia (GO), Brasil.

${ }^{4}$ Curso de Fisioterapia, Universidade Estadual de Goiás - UEG - Goiânia (GO) Brasil.

Conflito de interesses: não há 


\section{Introdução}

Os músculos do assoalho pélvico (MAP) apresentam função de sustentar os órgãos pélvicos e manter as funções fisiológicas de armazenamento e eliminação dos produtos de excreção da bexiga e do reto ${ }^{1}$. O levantador do ânus é um dos principais músculos do assoalho pélvico, sendo inervado pelo nervo pudendo e composto por fibras estriadas dos tipos I e $\mathrm{II}^{1}$. O risco de disfunções do assoalho pélvico como incontinência urinária, incontinência fecal e prolapsos genitais aumenta quando os MAP perdem sua integridade ${ }^{2}$.

Estudos mostraram que a maioria das disfunções do assoalho pélvico está associada a sinais eletromiográficos de desnervação da sua musculatura ${ }^{1,2}$. O parto vaginal é um dos fatores de risco para essa desnervação porque, durante o segundo estágio do trabalho de parto, a cabeça fetal pode provocar compressão e alongamento do nervo pudendo, causando estiramento dos tecidos e ligamentos da pelve $e^{3,4}$. Outros estudos demonstraram que o parto vaginal pode diminuir a força muscular do assoalho pélvico de mulheres primíparas e multíparas, quando comparadas com mulheres submetidas à cesárea e nulíparas ${ }^{4-6}$.

A incontinência urinária é descrita como a disfunção do assoalho pélvico mais prevalente, afetando 34 a 38\% de primíparas e multíparas três meses após o parto ${ }^{7}$. Para prevenir e tratar a incontinência urinária, estudos mostraram que o treinamento dos MAP por meio de exercícios é eficaz, pois reduz a perda involuntária de urina e aumenta a força muscular ${ }^{8-10}$. O treinamento com exercícios para os MAP é uma intervenção simples e de baixo risco ${ }^{11}$. O período de pós-parto tardio é considerado ideal para realizar programas de exercícios para promover a continência urinária e prevenir a incontinência urinária ${ }^{8-10}$.

O ganho de força muscular com o treinamento dos MAP pode ser medido, utilizando-se vários métodos. Um dos métodos mais utilizados na prática clínica é o da palpação vaginal digital, em que a força muscular é graduada segundo diferentes escalas ${ }^{12}$. Outros métodos utilizados são a manometria e a perineometria, que registram a pressão exercida pela contração vaginal e, assim, de forma indireta, indicam a força dos $\mathrm{MAP}^{13}$.

Há poucos estudos sobre os efeitos do treinamento dos MAP de puérperas que demonstraram a melhora da força muscular e a prevenção de incontinência urinária no pós-parto ${ }^{7,9,14-16}$. Esses estudos não apresentaram uma abordagem individualizada e sistematizada de treinamento ${ }^{10}$.

Após revisão da literatura não foi identificado um programa de exercícios padronizado para a prevenção de disfunções e melhora da força dos MAP em puérperas multíparas. Nesse sentido, a padronização de um protocolo individualizado e supervisionado de exercícios é de grande relevância, uma vez que mulheres submetidas ao parto vaginal constituem uma população de risco para disfunções do assoalho pélvico ${ }^{3}$, principalmente aquelas com história de outros partos vaginais ${ }^{17}$.

Nesse contexto, os objetivos deste estudo foram investigar o efeito de um programa individualizado e supervisionado de exercícios para o fortalecimento dos MAP no pós-parto de multíparas e verificar a correlação entre a palpação vaginal digital e o perineômetro.

\section{Métodos}

\section{Participantes}

Conduziu-se um ensaio clínico aberto na Maternidade Pública Edgar Carneiro de Souza e em duas Unidades Básicas de Saúde da cidade de Jataí, Goiás, entre janeiro e julho de 2011.

Foram incluídas mulheres com idade entre 18 e 35 anos, paridade entre 2 e 4 filhos, que se encontravam no pós-parto vaginal imediato, de feto a termo (parto atual e anteriores), sem queixas de sintomas urinários antes e durante a gestação e até 40 dias após o parto. Foram excluídas mulheres com antecedente de parto vaginal instrumental, cesárea, parto gemelar, antecedente de peso fetal $<2.500 \mathrm{~g}$ ou $>4.000 \mathrm{~g}$, cirurgia perineal e que declararam realizar exercícios perineais.

Mulheres que se encontravam na maternidade e que aguardavam alta hospitalar após o parto e que preenchiam os critérios de inclusão e exclusão foram convidadas a participar do estudo. A puérpera que concordava em participar, após o esclarecimento de todas as dúvidas, assinava o termo de consentimento livre e esclarecido. A seguir, agendou-se um retorno, entre o $35^{\circ}$ e $40^{\circ}$ dia de puerpério, para uma das Unidades Básicas de Saúde integrante do estudo. A unidade de saúde foi determinada pela puérpera de acordo com a proximidade do seu domicílio.

No total, 40 puérperas foram consecutivamente convidadas a participar do estudo. Dessas, 16 foram excluídas por não comparecerem ao retorno à unidade básica de saúde. O não comparecimento ocorreu por diversos motivos, tais como não ter com quem deixar seus outros filhos $(n=2)$, mudança de domicílio $(n=5)$, retorno à atividade laborativa $(\mathrm{n}=3)$. Outras puérperas não foram encontradas nos telefones e endereços informados $(n=6)$. No decorrer do estudo, uma participante retirou o consentimento. Dessa forma, a amostra do estudo foi constituída por 23 participantes distribuídas em dois grupos: Grupo Intervenção (GI) e Grupo Controle (GC). A inclusão da primeira participante em um dos grupos foi determinada por sorteio, sendo designada para o GI. A inclusão das demais participantes ocorreu de forma alternada entre o GC $(n=12)$ e o GI $(n=11)$.

No retorno entre o $35^{\circ}$ e $40^{\circ}$ dia após o parto, as participantes foram submetidas à primeira avaliação, incluindo 
entrevista sobre dados sociodemográficos, antecedentes obstétricos, presença de sintomas urinários, conhecimento sobre exercícios perineais e exame físico. Foi mensurada a força dos MAP por meio de dois métodos: palpação vaginal digital e perineômetro. Além disso, as puérperas do GI iniciavam o programa de exercícios para os MAP. As participantes do GC não recebiam orientação quanto a exercícios e eram agendadas para retorno ao final de oito semanas, quando ocorreu a segunda avaliação.

Ao final do programa de exercícios, conduziu-se a segunda avaliação. Nesse momento, a puérpera foi questionada quanto à presença de algum sintoma urinário e a força dos MAP foi aferida novamente por meio dos dois métodos.

A média de idade das participantes do GI foi de $24 \pm 4,5$ anos e do GC foi de 25,3 $\pm 4,03$ anos ( $p=0,4)$. Não houve diferença entre os grupos quanto à raça/etnia $(\mathrm{p}=0,3)$, estado marital $(\mathrm{p}=0,3)$, escolaridade $(\mathrm{p}=0,1)$, renda familiar $(\mathrm{p}=0,6)$, peso da puérpera $(\mathrm{p}=0,3)$, paridade $(\mathrm{p}=0,6)$, prática de atividade física $(\mathrm{p}=0,2)$, ocorrência de episiotomia $(\mathrm{p}=0,1)$, ou laceração perineal $(\mathrm{p}=0,3)$ e peso do recém-nascido $(\mathrm{p}=0,1)$.

Este estudo foi aprovado pelo Comitê de Ética em Pesquisa Humana e Animal do Hospital das Clínicas da Universidade Federal de Goiás.

\section{Sintomas urinários}

Para investigar a presença de sintomas de incontinência urinária de esforço utilizou-se o International Consultation on Incontinence Questionnaire - Short Form $(\mathrm{ICIQ}-\mathrm{SF})^{18}$, traduzido e validado para a língua portuguesa do Brasil ${ }^{19}$. Esse questionário é composto de três questões que avaliam a frequência de perdas urinárias, a gravidade das perdas e o impacto da incontinência urinária na qualidade de vida. Além disso, há um conjunto de oito itens de autodiagnóstico relacionados às causas ou às situações de incontinência urinária vivenciadas pelos pacientes ${ }^{19}$. O escore do questionário consiste na soma dos resultados das 3 questões e varia de 0 a 21 pontos. Quanto maior o escore, maior o impacto da incontinência na qualidade de vida ${ }^{19}$. Para este estudo, não foi considerada a terceira questão sobre o impacto da incontinência na qualidade de vida e, portanto, o escore não foi calculado. As demais questões foram utilizadas para verificar a ocorrência de sintomas de incontinência urinária de esforço.

Além do questionário ICIQ-SF, foram elaboradas questões para investigar a existência de sintomas de urgência miccional, urge-incontinência, polaciúria e noctúria. Para verificar a presença de urgência foi questionado sobre uma "vontade urgente e incontrolável de urinar"; para a urge-incontinência questionou-se sobre uma "perda de urina associada a forte vontade de urinar"; para a polaciúria perguntou-se se a participante "urina frequentemente durante o dia" e para a noctúria, se a participante "urina frequentemente durante a noite".

\section{Medida da força muscular}

A força dos MAP foi medida com a participante em posição ginecológica, abdômen, assoalho pélvico e membros inferiores desnudos. Para cada método de avaliação da força muscular do assoalho pélvico, a participante realizou três contrações com intervalo de um minuto entre uma e outra. Para a palpação vaginal digital, considerou-se a contração que atingiu o maior valor ou contração máxima. Para o perineômetro, calculou-se a média das três contrações.

A palpação vaginal digital foi realizada com a introdução dos dedos indicador e médio a aproximadamente seis centímetros do introito vaginal, solicitando-se a contração dos MAP. A força dos MAP foi classificada de acordo com a escala de $O x$ ford modificada $^{12}$ — grau 0: ausência de contração muscular; grau 1: esboço de contração muscular não sustentada; grau 2: presença de contração de pequena intensidade, mas que se sustenta; grau 3: contração sentida, comprimindo os dedos do examinador com pequena elevação da parede vaginal posterior; grau 4: contração satisfatória, que aperta os dedos do examinador com elevação da parede vaginal posterior em direção à sínfise púbica; grau 5: contração forte, compressão firme dos dedos do examinador com movimento positivo em relação à sínfise púbica ${ }^{12}$.

Após 15 minutos de repouso, realizou-se a avaliação da força dos MAP por meio do perineômetro (Peritron ${ }^{\mathrm{TM}}$ 9300V-Cardio-Design, Austrália. Esse dispositivo possibilita a aferição, de forma indireta, da força de contração, uma vez que registra a pressão exercida pela contração perineal. Esse registro é dado em centímetros de água $\left(\mathrm{cmH}_{2} \mathrm{O}\right)^{13}$. A sonda vaginal foi introduzida, após ser revestida por preservativo sem lubrificante, a três centímetros do introito vaginal.

\section{Programa de exercícios}

O programa de treinamento foi elaborado com base em outros programas e diretrizes de exercícios para os MAP $^{11,20,21}$ e adaptado para este estudo. Foi realizado individualmente com frequência de duas vezes por semana, por oito semanas consecutivas, com duração de $40 \mathrm{minutos} / \mathrm{sessão,} \mathrm{em} \mathrm{uma} \mathrm{sala} \mathrm{da} \mathrm{Unidade} \mathrm{Básica}$ de Saúde. O programa consistiu de exercícios resistidos para os MAP realizados nas posições de decúbito dorsal, lateral e ventral, quatro apoios, sentada e de pé.

Antes de iniciar cada sessão de exercícios era realizado um aquecimento, que consistiu em alongamentos dos membros inferiores e coluna lombar, seguido de mobilização pélvica (exercícios ativos de anteroversão, e retroversão pélvicas e inclinação pélvica). 
Além dos exercícios, na primeira sessão da primeira semana foi realizada uma instrução verbal sobre a anatomia do assoalho pélvico, as funções dos MAP e a importância dos exercícios após o parto para a prevenção de disfunções do assoalho pélvico. A instrução foi realizada utilizando-se pranchas de anatomia.

Todas as sessões foram iniciadas com a participante em decúbito dorsal e, nessa posição, uma das pesquisadoras associou a palpação vaginal digital à instrução verbal para a contração do assoalho pélvico, com o objetivo de verificar e melhorar, a cada sessão, a habilidade da participante em contrair e relaxar os MAP.

Os exercícios resistidos consistiam em contrações voluntárias máximas e mantidas dos MAP, seguidas de contrações rápidas. Essas contrações foram realizadas durante a expiração. As contrações máximas e mantidas foram associadas à contração do músculo transverso do abdômen, observada através de um leve movimento de tração da cicatriz umbilical na direção da coluna vertebral e de tração da cintura para dentro ${ }^{22}$.

Os exercícios foram realizados em 3 séries de 8 a 10 contrações máximas mantidas, inicialmente, por 10 segundos com intervalo de repouso de 10 segundos e, posteriormente, em 3 séries de 12 a 16 contrações máximas mantidas por 20 segundos com intervalo de 20 segundos de repouso. Cada contração máxima e mantida era seguida de contrações rápidas realizadas em três séries de três a cinco repetições, com duração de dois segundos de contração e seis segundos de repouso. O programa de exercícios detalhado está disponínel on-line ${ }^{23}$.

\section{Análise estatística}

Os resultados são apresentados em média e desvio padrão (DP), ou frequências absoluta e relativa de acordo com o tipo de variável. Para verificar a igualdade entre os grupos com relação aos dados sociodemográficos e obstétricos utilizaram-se os testes exato de Fisher e do $\chi^{2}$. Para comparar as alterações na variável quantitativa força muscular medida pelo perineômetro, utilizou-se o teste $t$ de Student. Para comparar as alterações na variável qualitativa ordinal força muscular medida pela palpação vaginal digital, utilizou-se o teste de Kolmogorov-Smirnov para duas amostras independentes. Para verificar a correlação entre os dois métodos de medida da força dos MAP, foi utilizado o coeficiente de correlação de Pearson. Considerou-se como significativo $\mathrm{p}<0,05$. Todas as análises foram realizadas utilizando-se o programa SPSS, versão 17.0.

\section{Resultados}

$\mathrm{Na}$ primeira avaliação, não foi observada diferença entre os grupos quanto à força dos MAP, medida pelo perineômetro $(\mathrm{p}=0,1)$ e pela palpação vaginal digital $(\mathrm{p}=0,4) . \mathrm{Na} 2^{\mathrm{a}}$ avaliação, houve diferença significativa da força dos MAP entre os grupos quando medida por meio dos dois métodos $(\mathrm{p}<0,001)$, respectivamente, conforme apresentado nas Tabelas 1 e 2.

$\mathrm{Na}$ segunda avaliação, nenhuma das participantes do GI apresentou sintomas de incontinência urinária e três participantes do GC relataram sintomas de urgência miccional.

Os dois métodos de medida da força muscular apresentaram correlação significativa nas duas avaliações ( $1^{\mathrm{a}}$ avaliação: $\mathrm{r}=0,8, \mathrm{p}<0,001 ; 2^{\mathrm{a}}$ avaliação: $\mathrm{r}=0,9, \mathrm{p}<0,001$ ).

Tabela 1. Comparação da força muscular do assoalho pélvico, medida por meio do perineômetro, entre mulheres que realizaram (Grupo Intervenção) e que não realizaram (Grupo Controle) o programa de exercícios

\begin{tabular}{|c|c|c|c|}
\hline Grupos & n & $\begin{array}{c}\text { Força muscular }\left(\mathrm{cmH}_{2} 0\right) \\
\text { Média } \mathrm{DPP}^{-}\end{array}$ & Valor $p$ \\
\hline $1^{a}$ avaliaç̦ão & & & 0,1 \\
\hline Grupo Intervenção & 11 & $37,5 \pm 11,9$ & \\
\hline Grupo Controle & 12 & $28,7 \pm 12,4$ & \\
\hline $2^{a}$ avaliação & & & $<0,001$ \\
\hline Grupo Intervenção & 11 & $52,1 \pm 12,8$ & \\
\hline Grupo Controle & 12 & $29,2 \pm 12,3$ & \\
\hline
\end{tabular}

Tabela 2. Comparacão da força muscular do assoalho pélvico, medida pela palpaccão vaginal digital (escala de 0xford modificada), entre mulheres que realizaram (Grupo Intervenção) e que não realizaram (Grupo Controle) o programa de exercícios

\begin{tabular}{|c|c|c|c|}
\hline \multirow{2}{*}{ Força muscular } & Intervenção & Controle & \multirow{2}{*}{ Valor $p$} \\
\hline & $n(\%)$ & n (\%) & \\
\hline $1^{a}$ avaliação & & & 0,4 \\
\hline Grau 2 & $2(18,1)$ & $3(25,0)$ & \\
\hline Grav 3 & $4(36,4)$ & $8(66,7)$ & \\
\hline Grau 4 & $5(45,5)$ & $-(0,0)$ & \\
\hline Grau 5 & $-(0,0)$ & $1(8,3)$ & \\
\hline Total & $11(100,0)$ & $12(100,0)$ & \\
\hline $2^{a}$ avaliação & & & $<0,001$ \\
\hline Grau 2 & $-(0,0)$ & $3(25,0)$ & \\
\hline Grau 3 & $\cdot(0,0)$ & $8(66,7)$ & \\
\hline Grav 4 & $5(45,5)$ & $-(0,0)$ & \\
\hline Grau 5 & $6(54,5)$ & $1(8,3)$ & \\
\hline Total & $11(100,0)$ & $12(100,0)$ & \\
\hline
\end{tabular}

Teste de Kolmogorov-Smirnov para duas amostras.

\section{Discussão}

Neste estudo, o grupo submetido ao programa de exercícios melhorou significativamente a força muscular quando comparado ao Grupo Controle. Outros estudos mostraram a melhora da força dos MAP de puérperas após um programa de exercícios ${ }^{7,9,14-16}$.

O programa de exercícios para os MAP realizado no presente estudo enfatizou a realização de poucas repetições diárias, com aumento gradativo da intensidade da força 
e do tempo de contração para o recrutamento do maior número de unidades motoras. Essa conduta é importante para o ganho de força muscular ${ }^{11}$. Assim, os programas de treinamento dos MAP podem provocar alterações musculares devido à adaptação neural durante as primeiras seis a oito semanas ${ }^{22}$.

Contudo, o sucesso dos exercícios para os MAP depende não somente de treinamentos mais intensivos, mas também da supervisão de um fisioterapeuta habili$\operatorname{tado}^{20,24}$. Esse profissional proporcionará um tratamento com empenho, o que ajudará na motivação e adesão ao programa de treinamento ${ }^{11,20}$. Neste estudo, observou-se uma boa adesão ao programa de exercícios, pois somente uma puérpera desistiu da participação no treinamento, alegando dificuldades em se deslocar até a unidade de saúde. Nenhuma das demais participantes do GI faltou a mais de quatro sessões consecutivas e a média de sessões realizadas foi 11 .

$\mathrm{Na}$ segunda avaliação, nenhuma das participantes do GI apresentou sintomas de incontinência urinária e três participantes do GC relataram sintomas de urgência miccional. Esse resultado sugere que o programa de treinamento foi efetivo para a prevenção da incontinência urinária pós-parto. O aumento da força dos MAP e, consequentemente, da continência urinária através do programa de treinamento, é devido à ativação do esfíncter uretral e à promoção de melhor suporte do colo vesical, estimulando uma contração forte e rápida dos MAP durante um aumento súbito da pressão intra-abdominal, que previne a descida da uretra e promove seu fechamento ${ }^{21,22}$.

O presente estudo associou a contração dos MAP à contração do músculo transverso do abdômen com o objetivo de intensificar a ação dos MAP. Outro estudo demonstrou que, com o relaxamento do músculo transverso do abdômen, há uma diminuição da atividade eletromiográfica dos MAP e que, com a contração desse músculo, há uma coativação dos $\mathrm{MAP}^{25}$. Além disso, realizou-se a respiração diafragamática e a expiração durante as contrações dos MAP com o objetivo de aumentar a atividade eletromiográfica dos $\mathrm{MAP}^{20}$.

Neste estudo, no início de cada sessão do programa de exercícios, foi realizada uma solicitação verbal à puérpera para contrair os MAP. A solicitação era associada à palpação vaginal digital. Essa conduta é um biofeedback verbal $^{26}$. O biofeedback se refere ao uso de várias técnicas audiovisuais que informam ao paciente sobre a atividade dos músculos estriados, enquanto este tenta controlar a função desses músculos. Outro estudo comparou um programa de treinamento com exercícios associados a biofeedback verbal com exercícios associados a biofeedback visual $^{26}$. Os autores verificaram que o grupo com biofeedback visual apresentou melhores resultados de controle da força e seleção dos MAP. No entanto, revisão sistemática de ensaios clínicos randomizados não demonstrou superioridade do biofeedback visual sobre o biofeedback verbal ${ }^{27}$.

Os dois métodos de medida da força dos MAP apresentaram correlação significativa. Um estudo no qual se analisou a correlação entre as medidas de força muscular, realizadas por meio da palpação vaginal digital e pelo perineômetro, concluiu que a palpação vaginal é importante para ensinar a contração correta dos MAP, mas não é um método com boa reprodutividade ${ }^{28}$. Outros autores, no entanto, mostraram resultados semelhantes aos do presente estudo ${ }^{6,13,29,30}$. Assim, acredita-se que a palpação vaginal digital possa ser usada na prática clínica para medir a força dos MAP, uma vez que a maioria dos estudos mostrou boa correlação com o perineômetro.

Os achados do presente estudo devem ser interpretados, considerando-se alguns aspectos. $\mathrm{O}$ tamanho amostral, embora pequeno, possibilitou a identificação de associações significativas. Destaca-se o delineamento do estudo, um ensaio clínico, e a utilização das técnicas mais frequente empregadas para avaliação da força dos MAP.

Ressalta-se a importância deste estudo na prevenção primária de disfunções do assoalho pélvico, pois o treinamento das MAP pode ser utilizado como prática preventiva e tem indicação em algumas fases da vida das mulheres, como a gestação, o puerpério e o climatério ${ }^{21,31}$. Recomenda-se que, independentemente da presença de sintomas de incontinência urinária, é aconselhável que todas as mulheres façam um programa de exercícios de reabilitação do assoalho pélvico após o parto ${ }^{5,32}$.

Concluindo, o presente estudo mostrou que o programa de exercícios resultou em aumento significativo da força dos MAP em puérperas e contribuiu para a prevenção de incontinência urinária. Além disso, verificou-se uma correlação significativa entre as medidas da força dos MAP pela palpação vaginal digital e pelo perineômetro. Isso indica que a palpação vaginal digital pode ser utilizada na prática clínica, uma vez que é um método de baixo custo e que demonstrou correlação significativa com um método objetivo. 
1. Yiou R, Costa P, Haab F, Delmas V. Anatomie fonctionnelle du plancher pelvien. Prog Urol. 2009;19(13):916-25.

2. Herbruck LF. The impact of childbirth on the pelvic floor. Urol Nurs. 2008;28(3): 173-84.

3. Chaliha C. Postpartum pelvic floor trauma. Curr Opin Obstet Gynecol. 2009;21(6):474-9.

4. Marshall K, Walsh DM, Baxter GD. The effect of a first vaginal delivery on the integrity of the pelvic floor musculature. Clin Rehabil. 2002; 16(7):795-9.

5. Baytur YB, Serter S, Tarhan S, Uyar Y, Inceboz U, Pabuscu Y. Pelvic floor function and anatomy after childbirth. J Reprod Med. 2007;52(7):604-10.

6. Batista EM, Conde DM, Amaral WN, Martinez EZ. Comparison of pelvic floor muscle strength between women undergoing vaginal delivery, cesarean section, and nulliparae using a perineometer and digital palpation. Gynecol Endocrinol. $2011 ; 27(11): 910-4$.

7. Mørkved S, Bø K. Effect of postpartum pelvic floor muscle training in prevention and treatment of urinary incontinence: a one-year follow up. BJOG. 2000;107(8):1022-8.

8. Chiarelli P, Murphy B, Cockburn J. Acceptability of a urinary continence promotion programme to women in postpartum. BJOG. 2003; 11 10(2): 188-96

9. Meyer S, Hohlfeld P, Achtari C, De Grandi P. Pelvic floor education after vaginal delivery. Obstet Gynecol. 2001;97(5 Pt 1):673-7.

10. Hay-Smith J, Mørkved S, Fairbrother KA, Herbison GP. Pelvic floor muscle training for prevention and treatment of urinary and faecal incontinence in antenatal and postnatal women. Cochrane Database Syst Rev. 2009;(1):CD007471.

11. Berghmans LC, Bø K, Bernards N, Grupping-Morel M, Bluyssen N, Hendriks $E$, et al. Clinical practice guidelines for the physical therapy of patients with stress urinary incontinence. Urodin Uroginecol. 2003;6(1):1-14.

12. Laycock J, Jerwood D. Pelvic floor muscle assessment: the PERFECT Scheme. Physiotherapy. 2001;87(12):631-42.

13. Frawley HC, Galea MP, Phillips BA, Sherburn M, Bø K. Reliability of pelvic floor muscle strength assessment using different test positions and tools. Neurourol Urodyn. 2006;25(3):236-42.

14. Chiarelli $P$, Cockburn J. Promoting urinary continence in women after delivery: randomised controlled trial. BM. 2002;324(7348):1241.

15. Mørkved S, Bø K. The effect of post-natal exercises to strengthen the pelvic floor muscles. Acta Obstet Gynecol Scand. 1996;75(4):382-5.

16. Mørkved S, Bø K. The effect of postpartum pelvic floor muscle exercise in the prevention and treatment of urinary incontinence. Int Urogynecol J Pelvic Floor Dysfunct. 1997;8(4):217-22.

17. Lukacz ES, Lawrence JM, Contreras R, Nager CW, Luber KM. Parity, mode of delivery, and pelvic floor disorders. Obstet Gynecol. 2006; 107(6): 1253-60.

18. Avery K, Donovan J, Abrams P. Validation of a new questionnaire for incontinence: the International Consultation on Incontinence
Questionnaire (ICIQ). Abstract $n^{\circ} 86$ of the International Continence Society 31 st annual meeting. Seoul, Korea. Neurourol Urodyn. $2001 ; 20(4): 510-2$.

19. Tamanini JTN, Dambros M, D'Ancona CAL, Palma PCR, Netto Júnior NR. Validação para o português do International Consultation on Incontinence Questionnaire - Short Form (ICIQ-SF). Rev Saúde Pública. 2004;38(3):438-44.

20. Pereira SB, Silva JM, Pereira LC. Treinamento dos músculos do assoalho pélvico. In: Palma PC, editor. Urofisioterapia: aplicações clínicas das técnicas fisioterapêuticas nas disfunções miccionais e do assoalho pélvico. Campinas: Personal Link Comunicações; 2009. p. 347-54.

21. Sampselle CM, Miller JM, Mims BL, Delancey JO, Ashton-Miller $\mathrm{JA}$, Antonakos CL. Effect of pelvic floor muscle exercise on transient incontinence during pregnancy and after birth. Obstet Gynecol. 1998;91(3):406-12.

22. DiNubile NA. Strength training. Clin Sports Med. 1991;10(1):33-62.

23. Universidade Federal de Goiás [Internet]. Fisioterapia: quadro com a descrição de programa de exercícios. Jataí (GO); 2012 [citado 2012 Set 13]. Disponível em: <http://fisioterapia.jatai. ufg.br/pages/39330>

24. Zanetti MR, Castro RA, Rotta AL, Santos PD, Sartori M, Girão M. Impact of supervised physiotherapeutic pelvic floor exercises for treating female stress urinary incontinence. São Paulo Med J. 2007; 125(5):265-9.

25. Sapsford RR, Hodges PW. Contraction of the pelvic floor muscles during abdominal maneuvers. Arch Phys Med Rehabil. 2001;82(8):1081-8.

26. Burgio KL, Robinson JC, Engel BT. The role of biofeedback in Kegel exercise training for stress urinary incontinence. Am J Obstet Gynecol. 1986;154(1):58-64.

27. Berghmans LC, Hendriks HJ, Bø K, Hay-Smith EJ, de Bie RA, van Waalwijk van Doorn ES. Conservative treatment of stress urinary incontinence in women: a systematic review of randomized clinical trials. Br J Urol. 1998;82(2):181-91.

28. Bø K, Finckenhagen HB. Vaginal palpation of pelvic floor muscle strength: inter-test reproducibility and comparison between palpation and vaginal squeeze pressure. Acta Obstet Gynecol Scand. 2001;80(10):883-7.

29. Sanches PRS, Ramos JGL, Schmidt AP, Nickel SD, Chaves CM, Silva Junior DP, et al. Correlação do escore de Oxford modificado com as medidas perineométricas em pacientes incontinentes. Rev HCPA \& Fac Med Univ Fed Rio Gd do Sul. 2010;30(2):125-30.

30. Isherwood PJ, Rane A. Comparative assessment of pelvic floor strength using a perineometer and digital examination. BJOG. 2000; 107(8):1007-11.

31. Heit M, Mudd K, Culligan P. Prevention of childbirth injuries to the pelvic floor. Curr Womens Health Rep. $2001 ; 1(1): 72-80$.

32. Dumoulin C. Postnatal pelvic floor muscle training for preventing and treating urinary incontinence: where do we stand? Curr Opin Obstet Gynecol. 2006; 18(5):538-43. 